\title{
TWO THEOREMS IN GEOMETRIC MEASURE THEORY
}

\author{
BY HERBERT FEDERER ${ }^{1}$
}

\section{Communicated by J. Wermer, February 8, 1966}

The following two propositions give some new information about the structure of differentiable maps. We use the symbols $R^{m}$ and $H^{s}$ to designate $m$ dimensional Euclidean space and $s$ dimensional Hausdorff measure, respectively.

THEOREM 1. If $m>r \geqq 0$ and $k \geqq 1$ are integers, $Y$ is a normed real vectorspace, $f: R^{m} \rightarrow Y$ is $k$ times continuously differentiable, and

$$
S=R^{m} \cap\{x: \operatorname{dimim} D f(x) \leqq r\},
$$

then

$$
H^{r+(m-r) / k}[f(S)]=0 .
$$

TheoRem 2. If $f: R^{m} \rightarrow R^{n}$ is Lipschitzian, $r$ is an integer, $0 \leqq r \leqq m$, and

$$
T=R^{n} \cap\left\{y: H^{m-r}\left(f^{-1}\{y\}\right)>0\right\},
$$

then $H^{r}$ almost all of $T$ can be covered by a countable family of $r$ dimensional submanifolds of class 1 of $R^{n}$.

The first theorem optimally sharpens the results of [4], where the history of the problem is discussed; its proof uses a refinement of the key lemma in [3], which dealt with the case $r=0$. The second theorem is related to the coarea formulae obtained in [2] and [1]. Proofs of both theorems will appear in the author's book Geometric measure theory.

\section{REFERENCES}

1. J. E. Brothers, Integral geometry in homogeneous spaces, Trans. Amer. Math. Soc. (to appear).

2. Herbert Federer, Curvature measures, Trans. Amer. Math. Soc., 93 (1959), 418-491.

3. A. P. Morse, The behavior of a function on its critical set, Ann. of Math. 40 (1939), 62-70.

4. Arthur Sard, Hausdorff measure of critical images on Banach manifolds, Amer. J. Math. 87 (1965), 158-174.

\section{BROWN UNIVERSITY}

1 This work was supported in part by a research grant from the National Science Foundation. 